\title{
Limbal Stem Cell Deficiency Arising From Systemic Chemotherapy With Hydroxycarbamide
}

\author{
Xiaoyan Ding, MD, PhD ${ }^{\star}, \dagger$, Rachel J. Bishop, MD ${ }^{\ddagger}$, Alexandra A. Herzlich, BS, MD ${ }^{\star}$, Mrinali \\ Patel, BS $^{\star}, \S$, and Chi-Chao Chan, MD \\ ${ }^{*}$ Section of Immunopathology, Laboratory of Immunology, National Eye Institute, National Institutes of \\ Health, Bethesda, MD \\ $\dagger$ Zhongshan Ophthalmic Center, Sun Yet-sen University, Guangzhou, China \\ $\$ C l i n i c a l$ Branch, National Eye Institute, National Institutes of Health, Bethesda, MD \\ §Howard Hughes Medical Institute, Chevy Chase, MD
}

\section{Abstract}

Purpose-The purpose of this study was to report a case of limbal stem cell deficiency (LSCD) after systemic chemotherapy with hydroxycarbamide.

Methods-Clinical manifestations and pathology are detailed.

Results-We describe the case of a woman with sickle cell disease, who developed bilateral LSCD after treatment with hydroxycarbamide. Histologic examination confirmed the diagnosis of LSCD, revealing goblet cells, inflammatory cells, deposits of new collagen components, and neovascularization in the peripheral cornea. Matrix metalloproteinase-3, fibronectin, and collagen III were also detected in the lesions.

Conclusions-The systemic use of the antineoplastic drug, hydroxycarbamide, may cause severe LSCD. We recommend that a medication history, including that of cytotoxic drugs, be considered in evaluating LSCD.

\section{Keywords}

limbal stem cell; ocular surface disease; chemotherapy drugs; hydroxycarbamide

\begin{abstract}
Hydroxycarbamide, also known as hydroxyurea, is a cytotoxic antineoplastic drug used to treat hematologic disorders, including sickle cell disease. As the first agent approved by the Food and Drug Administration to prevent complications of sickle cell disease, hydroxycarbamide is currently the most commonly prescribed treatment for the disease. ${ }^{1}$ Recently, indications for its use have been expanded to include antiretroviral therapy for patients with human immunodeficiency virus. Reported side effects include vomiting, diarrhea, bone marrow toxicity, alopecia, liver dysfunction, and kidney dysfunction. To date, only a single case of ocular surface toxicity after the use of hydroxycarbamide has been reported in the literature, and this case involved limited tissue pathology. ${ }^{2}$ We herein describe a case of limbal stem cell deficiency (LSCD) after chemotherapy with hydroxycarbamide and also the ocular pathology findings confirming the diagnosis.
\end{abstract}

Reprints: Chi-Chao Chan, MD, Section of Immunopathology, Laboratory of Immunology, National Eye Institute, National Institutes of Health, Building 10, Room 10N103, 10 Center Drive, Bethesda, MD 20892-1857 (e-mail: E-mail: chanc@ @ei.nih.gov). 


\section{Case Report}

The patient is a 49-year-old African American woman with sickle cell disease, diagnosed at age 15 , who was treated with hydroxycarbamide, $500 / 1000 \mathrm{mg}$ alternating daily, for pulmonary hypertension from before 2001 until her death in 2007. Her other medications included oxycodone, meperidine, ranitidine, sildenafil, fexofenadine, olopatadine, albuterol, fluticasone propionate, and folic acid. She had no history of treatment with other chemotherapy agents.

Initial eye examination of this patient in 2001, at which point she had already began hydroxycarbamide treatment, revealed normal cornea in both eyes (OU). She continued systemic hydroxycarbamide therapy, and subsequent ocular examination 2 years later in May 2003 revealed pterygium-like peripheral corneal neovascularization in both eyes (Fig. 1, upper panels). Her vision at that time was 20/25 OU. Over the ensuing 4 years, her vision deteriorated to 20/50 right eye (OD) and 20/200 left eye (OS), and she experienced significant photophobia and ocular redness. Slit-lamp examination during this period revealed the development of inferior subepithelial neovascularization, which gradually extended into the central cornea bilaterally (Fig. 1, lower panels). Schirmer test showed $13 \mathrm{~mm}$ in right eye and $12 \mathrm{~mm}$ in left eye in June 2007. She was treated with topical prednisolone and cyclosporine, with variable response.

This patient had no history of ocular disease and had not been on any topical ocular medications. No dermal alterations had been found in the 5 years preceding this ocular disease, except a short-lived, mild rash when she first began taking hydroxycarbamide. She denied any family history of ocular diseases. The patient died of complications of sickle cell disease in July 2007.

Pathologic evaluation of the conjunctiva revealed pingueculae, loss of goblet cells with scar tissue formation, and infiltration of $\mathrm{CD} 45 \mathrm{RO}^{+}$and $\mathrm{CD} 20^{+}$inflammatory cells. In addition, neovascularization was present, most pronounced at the limbus. The central corneal epithelium was markedly thinned and irregular, with a neovascular pannus. Bowman layer was absent in the periphery and contained multiple small breaks centrally. T cells, B cells, and scattered macrophages infiltrated the corneal lesions, particularly along the vessels. Matrix metalloproteinase-3 (MMP-3), fibronectin, and collagen III were also detected in the lesions by immunohistochemistry (Fig. 2). Based on the clinical history and pathology findings, a diagnosis of LSCD secondary to hydroxycarbamide was made.

\section{Discussion}

LSCD is characterized by a cluster of ocular manifestations involving the cornea, including persistent or recurrent corneal epithelial defects, ulceration, chronic inflammation, corneal vascularization, scarring, and conjunctivalization, eventually resulting in corneal opacity. ${ }^{3}$ LSCD can occur focally or involve the entire limbus. ${ }^{4}$ Chronic instability of the corneal epithelium and ulceration may lead to progressive corneal thinning, with subsequent risk of perforation. Patients with LSCD commonly complain of severe photophobia and ocular irritation, in addition to profound vision loss.

LSCD results from a variety of acquired disorders, including Stevens-Johnson syndrome, chemical injuries, ocular cicatricial pemphigoid, and repeated surgeries or cryotherapy to the limbal region. ${ }^{3}$ Because of their propensity to target rapidly dividing cells, topical cytotoxic agents such as 5-fluorouracil and mitomycin $\mathrm{C}$ have also been recognized as inducers of epithelial defects and LSCD. 5,6 There is a case of LSCD described in the literature in which the patient developed recurrent epithelial defects after five 1-week courses of topical mitomycin C. ${ }^{5}$ However, only 1 case of epithelial erosion arising from systemic cytotoxic therapy has ever been reported. ${ }^{2}$ 
Systemic medications are introduced to the avascular cornea in very low levels through the tear film, aqueous humor, and/or limbal vasculature. We expect that this low penetrance of systemic medications to the cornea underlies the rarity of LSCD after systemic chemotherapy. Based on clinical findings in this patient, including normal Schimer test, the dry eye scenario seems unlikely in this case, although in most patients undergoing systemic chemotherapy, the development of dry eye is more likely than a direct effect to the limbus by the chemotherapeutic agent.

In general, most complications resulting from hydroxycarbamide seem to be reversible in the early stages, typically resolving after drug cessation. ${ }^{2}$ We therefore suggest that the diagnosis of LSCD should be considered in cases of corneal epitheliopathy of unknown etiology and that a medication history, including that of cytotoxic drugs, be explored. Once drug toxicity is suspected, cessation or modification of the toxic agent should be considered.

To our knowledge, this is the second reported case of hydroxycarbamine-induced LSCD and the first with detailed pathology. Interestingly, the superior limbus seems to be affected earlier than the inferior region in this case, which may reflect the fact that there are more active stem cells in the superior limbus. ${ }^{7}$ Although both cases demonstrate a link between systemic hydroxycarbamine therapy and LSCD, very little is currently understood about the mechanisms underlying this adverse effect. Further studies are needed to explore the mechanism of action and to identify factors that predispose individuals to this toxicity.

In addition to goblet cell loss, which is the classic pathologic finding in LSCD, this case presented with ocular surface inflammation, neovascularization, and new immature collagen deposition, as indicated by MMP-3, fibronectin, and collagen type III expression in the cornea. These new collagens produced by conjunctival fibroblasts may represent an attempt to replace the loss of limbal stem cells. Our findings therefore support the hypothesis that an epithelialmesenchymal transition of limbal epithelial progenitor cells constitutes the pathogenic basis of LSCD. The expression of MMP-3, fibronectin, and collage type III may also be related to inflammation and neovascularization found in the cornea.

Based on this finding, we hypothesize that aggressive anti-inflammatory therapy and treatment with matrix metalloproteinase inhibitors might help reduce the attrition of the remaining corneal epithelial cells and prevent the development of an LSCD-induced neovascular panus.

\section{Acknowledgements}

Supported by NEI Intramural Research Program.

\section{References}

1. Schechter AN, Rodgers GP. Sickle cell anemia-basic research reaches the clinic. N Engl J Med 1995;332:1372-1374. [PubMed: 7536300]

2. Ellies P, Anderson DF, Topuhami A, et al. Limbal stem cell deficiency arising from systemic chemotherapy. Br J Ophthalmol 2001;85:373-374. [PubMed: 11277104]

3. Ang LPK, Tan DTH. Ocular surface stem cells and disease: current concepts and clinical applications. Ann Acad Med Singapore 2004;33:576-580. [PubMed: 15531952]

4. Dua HS, Azuara-Blanco A. Limbal stem cells of the corneal epithelium. Surv Ophthalmol 2000;44:415-425. [PubMed: 10734241]

5. Dudney BW, Malecha MA. Limbal stem cell deficiency following topical mitomycin C treatment of conjunctival-corneal intraepithelial neoplasia. Am J Ophthalmol 2004;137:950-951. [PubMed: 15126170] 
6. Pires RT, Chokshi A, Tseng SC. Amniotic membrane transplantation or conjunctival limbal autograft for limbal stem cell deficiency induced by 5-fluorouracil in glaucoma surgeries. Cornea 2000;19:284287. [PubMed: 10832684]

7. Pellegrini G, Golisano O, Paterna P, et al. Location and clonal analysis of stem cells and their differentiated progeny in the human ocular surface. J Cell Biol 1999;145:769-782. [PubMed: 10330405] 

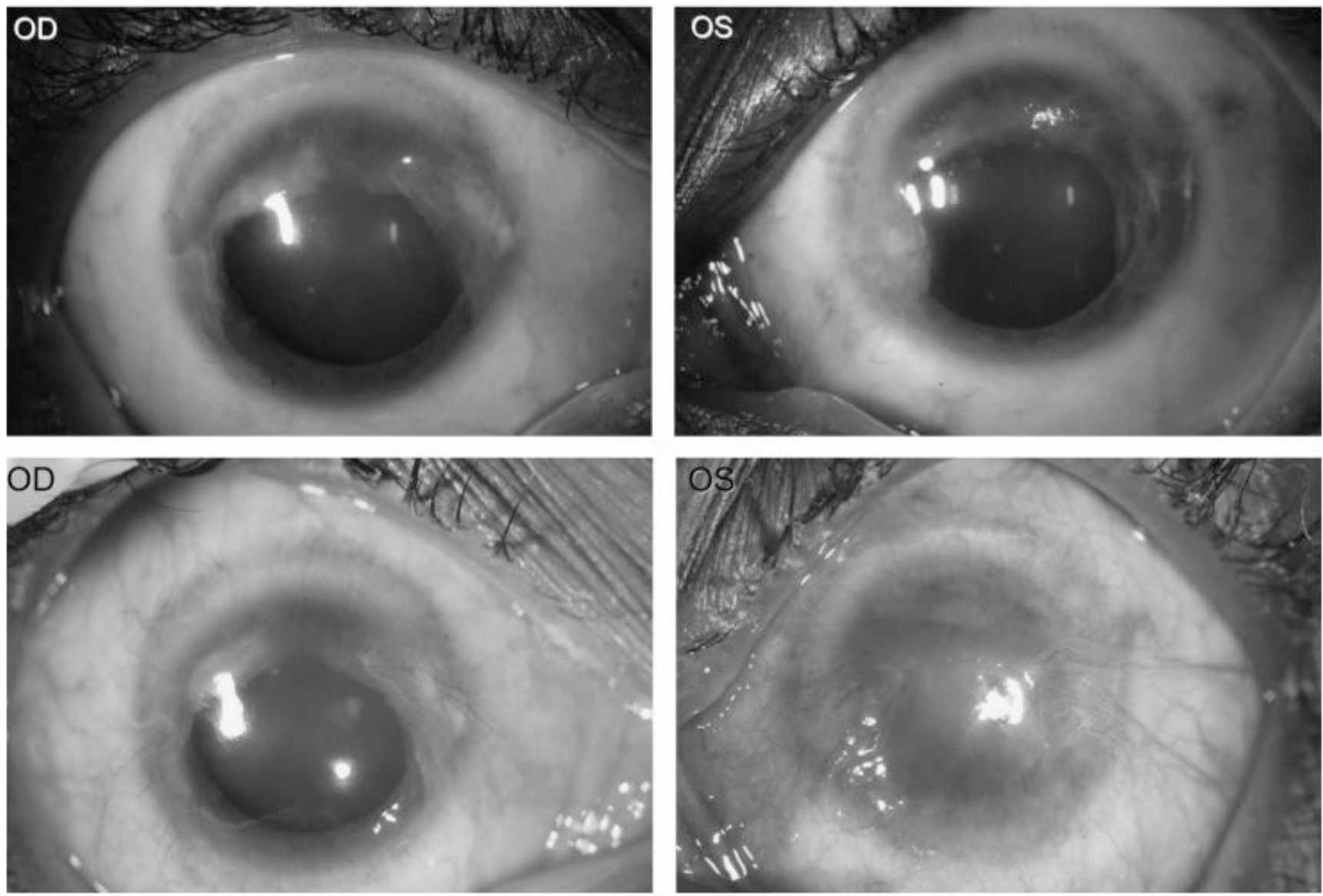

Figure 1.

Ocular photography in May 2003 (upper panels) and February 2007 (lower panels). Upper panel: Pterygium-like lesions cover 270 degree of the conjunctiva and peripheral cornea involving the nasal, superior, and temporal areas of both eyes. Lower panel: Progressive pterygium-like growths extend to the central and inferior cornea with neovascularization, more severe in the left eye than in the right eye. 


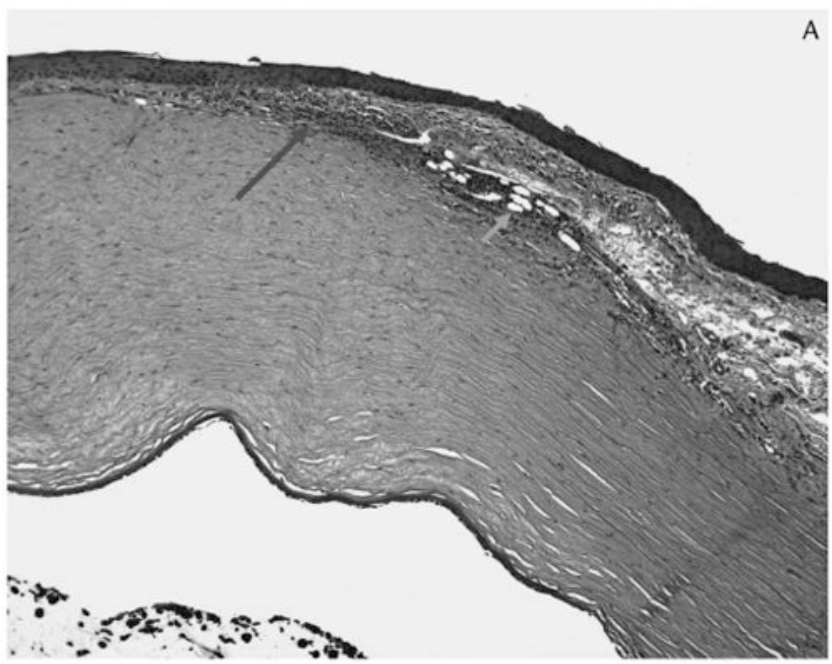

C

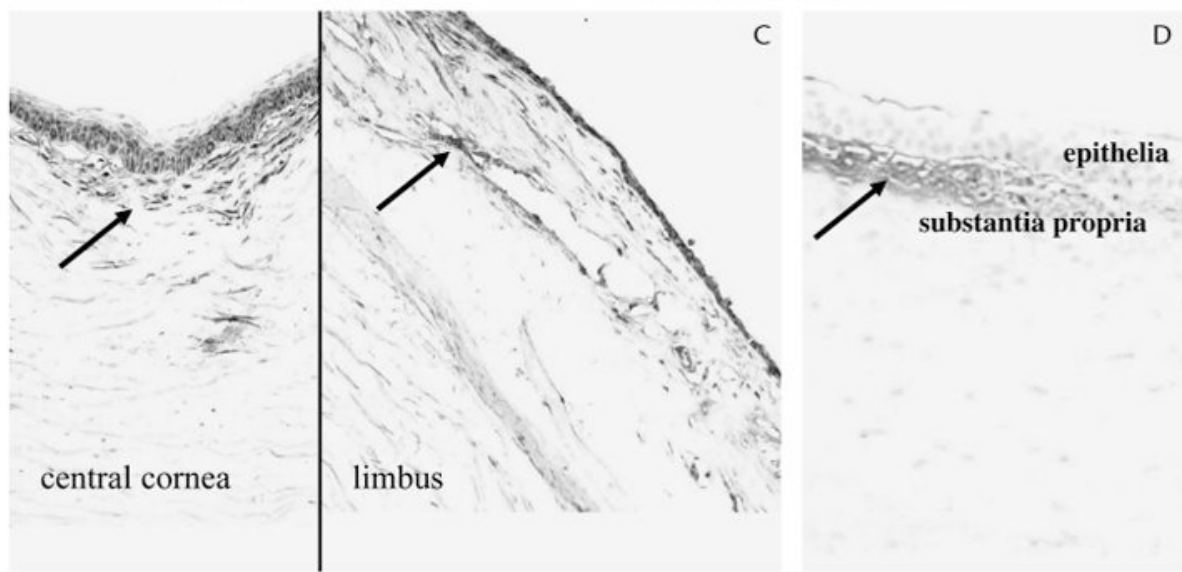

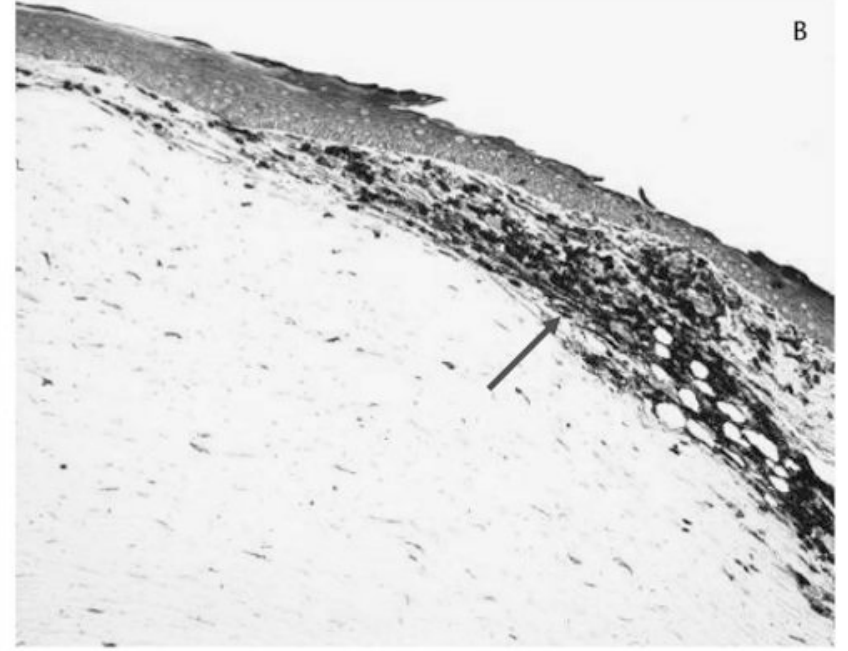

D

Figure 2.

Photomicrographs of the left eye. A, An aggregation of inflammatory cells (long arrow) and neovascular vessels (short arrow) is observed in the limbal cornea. B, The inflammatory cells are mainly T lymphocytes (arrow). C, MMP-3 (arrows) is detected in the limbus and central cornea. D, Fibronectin (arrow) is detected in the central cornea with abrasion. E, Collagen III (arrow) is detected in the limbus with neovascularization. (A, hematoxylin and eosin; B-E, avidin-biotin complex immunoperoxidase; $\times 100$.) 